\title{
Reliability Quantification of Advanced Stirling Convertor (ASC) Components
}

\author{
Ashwin R. Shah ${ }^{1}$ and Igor Korovaichuk ${ }^{2}$ \\ Sest, Inc., Middleburg Heights, Ohio 44130 \\ and \\ Edward Zampino ${ }^{3}$ \\ National Aeronautics and Space Administration, Glenn Research Center, Cleveland, Ohio 44135
}

\begin{abstract}
The Advanced Stirling Convertor, is intended to provide power for an unmanned planetary spacecraft and has an operational life requirement of 17 years. Over this 17 year mission, the ASC must provide power with desired performance and efficiency and require no corrective maintenance. Reliability demonstration testing for the ASC was found to be very limited due to schedule and resource constraints. Reliability demonstration must involve the application of analysis, system and component level testing, and simulation models, taken collectively. Therefore, computer simulation with limited test data verification is a viable approach to assess the reliability of ASC components. This approach is based on physics-of-failure mechanisms and involves the relationship among the design variables based on physics, mechanics, material behavior models, interaction of different components and their respective disciplines such as structures, materials, fluid, thermal, mechanical, electrical, etc. In addition, these models are based on the available test data, which can be updated, and analysis refined as more data and information becomes available. The failure mechanisms and causes of failure are included in the analysis, especially in light of the new information, in order to develop guidelines to improve design reliability and better operating controls to reduce the probability of failure. Quantified reliability assessment based on fundamental physical behavior of components and their relationship with other components has demonstrated itself to be a superior technique to conventional reliability approaches based on utilizing failure rates derived from similar equipment or simply expert judgment.
\end{abstract}

\section{Introduction}

$\mathrm{T}$ HE Advanced Stirling Convertor (ASC) transforms the thermal energy from radio-isotope material into the electrical energy required to power spacecraft functions. Stirling convertor is a free-piston device with a limited number of moving parts, and the current design has eliminated all principal wear out and life limiting mechanisms at a certain design temperature. In order to ascertain reliability of the Advanced Stirling convertor and minimize associated risks during pre-launch handling, assent, space flight, and possible variation in operating temperature, it is critical to quantify and assure the reliability of its components and the overall system for a mission life of at least 17 years $^{1,2}$.

The approach used to quantify the reliability of Advanced Stirling Convertor components is based on the consideration of physical variables and their distributions. The application of the technique is aimed at the objective of assuring availability of power for 17 years with desired performance and efficiency. The very large test time and the cost of a large sample of test units, means that life testing of the ASC units is very limited. Therefore, computer simulation with limited test data verification is a viable approach to assess the reliability of ASC components. Physics-based reliability analysis involves the relationship among the design variables based on physics, mechanics, material behavior models, interaction of different components and their respective disciplines such as structures,

\footnotetext{
${ }^{1}$ President, 18101 Jefferson Park Road Suite 104, AIAA Lifetime member.

${ }^{2}$ Aerospace Engineer, 18101Jefferson Park Road Suite 104.

${ }^{3}$ Reliability Engineer.
} 
materials, fluid, thermal, mechanical, electrical, etc. Additionally, these models are based on the available test data, which can be updated, and analysis be refined as more data and information becomes available. The failure mechanisms and causes of failure are included in the analysis, especially in light of the new information, in order to develop guidelines to improve design reliability and better operating controls to reduce the probability of failure. Quantified reliability assessment based on fundamental physical behavior of components and their relationship with other components is a superior technique to conventional reliability approaches based on utilizing failure rates derived from similar equipment or simply expert judgment.

The convertor components such as heater head, displacer assembly including displacer spring, alternator assembly, fasteners, regenerator, gas bearings and pressure vessel are critical for the detailed physics-based reliability assessment. It identifies the possible failure modes of the critical convertor components and simulates their underlying failure mechanisms by means of analytical and computational models to evaluate the performance and failure criteria in terms of the design variables sensitivity. Identification of the governing design variables is dependent upon component architecture, material properties, fabrication process, and the life cycle environment. Life cycle environment includes storage, handling, flight mission, as well as the expected severity and duration of the load conditions for each stage. Load conditions include various temperature profiles and pressure levels, vibration and/or shock loads, radiation levels, and loads caused by operational parameters such as current and voltage.

The probabilistic response models combined with the design variables test data are used to predict the reliability of convertor components based on the limit state concept. This concept requires a relationship between the underlying physics of failure, material models, design variables, and test data. Due to the complexity of the geometry and material models for convertor components, it was necessary to apply high-level computational tools such as non-linear finite element analysis, computational fluid dynamics and fast probability integration for the reliability assessment. One of the challenges during simulation is the prediction of variation in material properties due to environmental changes and aging effect. The two greatest major factors contributing to the material aging of components are high temperature and gigacycle loading. The quality of the convertor reliability prediction strongly depends on adequacy of life prediction models such as high temperature material creep and gigacycle fatigue.

Additionally, the sensitivity of the components reliability to the design variables has been described and quantified. The significance of this approach is that it directly relates to the design changes required to improve the reliability.

\section{Probabilistic Analysis Method for the ASC components}

The ASC consists of many components which may fail due to a variety of failure mechanisms related to structural loads and weaknesses, thermal energy, fluid flow, electro-magnetic effects, etc.. Theoretically, all potential failure modes must be considered when predicting the reliability of convertor components. However, preliminary evaluations suggested that the failure probability of some identified failure modes is so low that their contribution to the system and component reliability is insignificant and were omitted from the assessment. Thus, it was sufficient to include only critical failure modes that dominantly contribute to the convertor reliability. Conventionally, one can identify the following convertor components associated with these failure modes:

1) Components exposed to high temperature such as the thin-walled heater head and the displacer dome. Creep rupture for the heater head and creep rupture and fatigue under high temperature and reciprocating pressure for the displacer dome are considered as dominant failure mechanisms.

2) Moving components which include displacer assembly and displacer spring attached to the flex rod and piston assembly. Moving components reciprocate axially with a frequency of about $100 \mathrm{~Hz}$ which impose more than 55 billion cycles during the 17 yrs of design mission life span. Therefore, giga cycle fatigue is the dominant failure mode for the moving components.

3) Joints provide structural integrity of the convertor components. They include a variety of brazes, welds, structural fasteners, and adhesives used to secure the convertor's various internal components. For the convertor reliable performance, no joints failure is desired.

4) Gas bearings are designed to provide smooth frictionless conditions for the axially reciprocating moving components. At GRC, a complex fluid dynamic computational analysis are being conducted to evaluate the convertor gas bearings performance under regular mission conditions. During launch, exposure of the convertor to random vibration may cause lateral interference of the convertor internal components. In the random vibration reliability effort, the lateral response of the gas bearings is attempted to simulate using the stiffness and damping coefficients, which were determined from the GRC fluid analysis. 
It must be stated that many of the failure modes that have been studied were identified as single point failures (SPFs), which means that failure of an individual component may lead to the convertor failure. These failure modes were classified as critical and most efforts were directed toward the reliability quantification of these components.

The general probabilistic procedure developed for the Stirling Convertor combines numerical simulations and the results of short and long term tests of particular components or material test articles. These tests were performed at the NASA Glenn Research Center. Long term component tests simulating the mission environment as well as loading and material process effects are required to quantify these uncertainties with a reasonable level of confidence. Due to cost and time constraints as well as technical limitations of simulating the space environment, it is not possible to run full-duration, long term tests that simulate the actual operating conditions. Additionally, it is difficult to capture the effects of design variable uncertainties in a limited number of tests. Therefore, computer simulations (virtual experiments) for different design and operating conditions, validated with limited test data, is a viable approach to understand the long-term behavior of components and aid quantification of the reliability. It also enables engineers to quantify the impact of variations in critical design variables on component performance reliability. The computer simulations can be validated with the available test data, and the theoretical and empirical system performance models can be enhanced to consider interactions among components and the applicable design variables.

A finite element method was used as an analysis tool to generate the performance and response functions necessary for the probabilistic analysis. In this procedure, it was critical to apply adequate material models describing the material behavior properly in accordance with the results of the testing.

This paper describes the reliability assessment of the convertor components contributed to various single point failure modes. Some of these major failure modes are: (i) creep of the hot section heater head; (ii) long-term fatigue of the displacer spring; (iii) leakage of the feed-throughs; (iv) components interference due to launch random vibration.

\section{A. Heater Head Reliability Assessment}

Since the fine grain Nickel based super-alloy has been selected for the heater head material, the efforts were focused on development of the creep response model. For that purpose, a model similar to the previously developed model by Oak Ridge National Laboratory ${ }^{2}$ for Inconel material was used. In order to capture different levels of temperatures and stresses, the equation for the "master" creep curve in the form below is used:

$$
\varepsilon^{*}=\exp \left[\gamma\left(t^{*}-1\right)\right]\left(t^{*}\right)^{\mathcal{S}}
$$

where $\varepsilon^{*}=\varepsilon / \varepsilon_{s s}$ and $t^{*}\left(=t / t_{s s}\right)$ are normalized creep strain and normalized time, respectively, and where $\varepsilon$ is the creep strain, $\varepsilon_{\mathrm{ss}}$ is the minimum creep strain at onset of tertiary creep. Additionally, $t$ is the elapsed time, and $t_{s s}$ is the time to onset of tertiary creep. The constants $\gamma$ and $\delta$ are material constants.

This simple equation approximates the creep curve with only two constants, both independent of heat treatment, stress, and temperature within the range of available test data. Variations in the behavior due to these factors are reflected by variations in $t_{s s}$ and $\varepsilon_{s s}$. The relationships between minimum creep strain rate, $\dot{\varepsilon}_{\mathrm{ss}}$ and time to onset of tertiary creep, $\mathrm{t}_{\mathrm{ss}}$ are given by the power law for the rupture time, $t_{R}$

$$
\dot{\varepsilon}_{s s}=B t_{R}^{\alpha}, t_{s s}=A t_{R}^{\beta}
$$

where $B, \alpha, \mathrm{A}$, and $\beta$ are material constants derived from the test data. The rupture time is approximated by the following equation:

$$
\log t_{R}=A_{0}+A_{1} \log \sigma+A_{2}(\log \sigma)^{2}+A_{3}(\log \sigma)^{3}+A_{4} T \log \sigma
$$

Coefficients $A_{0}, A_{1}, A_{2}, A_{3}$, and $A_{4}$ are determined from the test performed at different temperatures, $T$ and stress, $\sigma$ levels.

1. Uncertainties in heater head design variables

In order to quantify reliability of the ASC heater head, a probabilistic analysis that accounts for uncertainties in the geometry, working fluid pressure, stud pressure (maintaining the GPHS (General Purpose Heat Source) contact with the heat collector plate), and material properties has been performed using non-linear finite element analysis for time dependent creep computation. All the design variables listed in Table I are used in the finite element analysis 
to compute creep strains and resulting rupture life. The probabilistic analysis simulates uncertainties and distributions used for each variable listed in Table 1.

Uncertainties in the heater head geometry are selected such that the bounds on the fabrication tolerances, per available drawings, do not exceed $\pm 0.05 \mathrm{~mm}$ in the dome section and $\pm 0.025 \mathrm{~mm}$ in the thin cylindrical section and relate to \pm 3 standard deviations, e.g. $0.0166 \mathrm{~mm}(0.05 \div 3)$ is the maximum standard deviation in the dome section.

The uncertainty in the helium working fluid pressure, assumed based on engineering judgment and discussions with design engineers, is such that the maximum expected variation in the pressure amplitude is $18 \%$ and corresponds to \pm 3 standard deviations. Uncertainty in the internal pressure is applied to the amplitude portion of the

Table 1. Uncertainties in the heater head design variables

\begin{tabular}{|l|c|c|l|}
\hline \multicolumn{1}{|c|}{ Random Design Variable } & $\begin{array}{c}\text { Mean } \\
\text { Value }\end{array}$ & $\mathrm{CoV}, \%$ & $\begin{array}{l}\text { Probability } \\
\text { Distribution }\end{array}$ \\
\hline Gas Pressure Amplitude, $M P a$ & - & 6.00 & Normal \\
\hline Stud Pressure, $M P a$ & - & 1.17 & Normal \\
\hline Minimum wall thickness, mm & - & 1.15 & Lognormal \\
\hline Material creep parameter, $A_{0}$ & - & 0.57 & Normal \\
\hline
\end{tabular}
pressure only while the mean value of cyclic working fluid pressure remains unchanged (assuming that the controller will adjust the mean pressure instantaneously).

Quantifying uncertainties in the material creep behavior is difficult due to the limited availability of test data for MarM-247 material. A review of the available data indicates that there were no more than two tests performed for a given cast or grain size at a given stress and temperature condition. Several different approaches and methodologies were adopted to quantify the uncertainties in the material creep behavior, however, important related aspects are described and only the final results are presented in this report. The complete description and quantification of the uncertainties in the material behavior involves quantifying uncertainties in all the coefficients of the master curve model (Eqs. 1 through 4). Also, it is worthy to note here that all these coefficients are correlated in a probabilistic sense. A large amount of test data is needed to quantify the uncertainties and cross covariance matrices among these coefficients. To circumvent the need for a large amount of test data, an engineering approach has been adopted based on the assumption that all these coefficients are fully correlated and all the uncertainties can be lumped into only one parameter $A_{0}$ in Eq. 4, which is more or less reflective of the material behavior. The scatter in the parameter, $A_{0}$ related to material creep behavior uncertainties is $1.72 \%$. Referring to Eq. 4 , it is clear how sensitive the rupture life is to this material constant. A small variation in the constant may cause large variation in the creep durability.

2. Reliability quantification of heater head at $850^{\circ} \mathrm{C}$

Full-fledged probabilistic analysis of heater head creep durability was performed for $850{ }^{\circ} \mathrm{C}$ service temperature. This analysis involved the following steps:

- Non-linear time-dependent finite element creep analysis simulations with uncertainties in the material behavior, pressure, stud force, and geometry (as listed per Table 1).

- Probability integration to predict uncertainties in the accumulated creep strain at critical locations. The advanced mean value (AMV) with iterations method was used to compute the probability distribution of the accumulated creep. Use of an iterative procedure alleviates the probability convergence issues due to non-linear behavior.

- Creep reliability was quantified using the following design criteria:

$$
\operatorname{Rel}\left(t_{\text {ser }}\right)=\operatorname{prob}\left[\left\{\varepsilon_{\text {des }}-\varepsilon_{\text {acc }}\left(t_{\text {ser }}\right)\right\}<0\right]
$$

where $\varepsilon_{d e s}$ and $\varepsilon_{a c c}$ are the critical creep design value and the creep strain accumulated during the service life span.

- A fast probability integration simulation was performed. Both local and average through-thickness criteria were investigated for the reliability assessment of the heater head. It was revealed that the location of maximum creep varies with the service time. Initially, after $1000 \mathrm{hrs}$, the maximum creep occurs near the interface with the heat collector. As the service time increases, the location of maximum creep shifts to the inner side of the dome where the working fluid pressure is applied. 


\section{Summary of the heater head reliability results}

It is worth noting here that the reliability analysis performed is based on the time to onset of tertiary creep limit state, and, thus, additional margin in life is available during the tertiary creep stage before actual rupture failure occurs. The creep life in the tertiary zone could be up to $30 \%$ of total rupture life. The analysis does not account for the effects of temperature reduction due to decay in the radioisotope material during the mission life. This decay could increase life by reducing the hot-end temperature or improve reliability for a given life requirement. Based on this, the following reliability of the heater head is predicted:

1) The heater head has at least a reliability of 0.99 for 17 yrs of life and reliability greater than 0.999 for 14 yrs life for the given creep criteria at a service temperature of $850^{\circ} \mathrm{C}$.

2) Uncertainties in the material creep behavior represented by parameter $\mathrm{A}_{0}$ should be reduced through qualitycontrol during material processing, heat treatment, inspection and testing in order to improve the reliability of the heater head.

\section{B. Reliability Assessment of Other Hot Components}

The displacer dome and hot cylinder are another two critical convertor components subject to elevated temperature in the range from $650{ }^{\circ} \mathrm{C}$ to $840{ }^{\circ} \mathrm{C}$. The displacer dome is a part of the convertor moving components designed to provide smooth reciprocal motion during the convertor life. The interaction of the moving components with other convertor components is realized by bearing capacity of the working gas which aims to maintain a proper gap allowing smooth frictionless sliding. Due to very tight clearances, gas high temperature controls the gas bearing capacity.

The reliability of the displacer dome is governed by the high temperature creep, giga cycle fatigue of the thinwalled section under cyclic pressure, and structural integrity of the baffles (used as a protective thermal shield) brazed to the dome. The lack of historical data on creep and fatigue performance of materials with extremely low wall thickness, order of one to two tens of a millimeter, at elevated temperature reduces the ability to depend on known methods to assure reliability of the hot section components. Therefore, the creep and fatigue analyses was based on available creep and fatigue properties of the bulk material with adjustable corrections for the wall thickness.

\section{Fatigue strength evaluation}

The fatigue life curves at elevated temperature for extremely thin sheets with a limited number of grains through wall thickness made of any nickel-based alloy were are hard to find in open literature. Therefore, an assumption is made based on the study of fracture toughness of other thin metallic foils to derive the properties fir thin material. The traditional practice which assumes that the toughness is minimum for plane-strain condition and reaches maximum for plane-stress condition may not be correct. A bell-shape curve may exist for metals and alloys with thickness below $0.30 \mathrm{~mm}$ having toughness value even below plain-strain condition. Therefore, the lower bound of the strength corresponding to the plain-strain condition obtained from testing of the specimens with thickness of 1 $\mathrm{mm}$ may be misleading in estimation of the strength and fatigue of the thin components below the $0.200 \mathrm{~mm}$. This is possibly due to the effect of material microstructure on high frequency cyclic behavior at elevated temperatures.

Another addressed issue is giga-cycles fatigue. This is characterized by extremely high number of cycles, order of 55 billions, that a baffle will experience during 17 yrs of mission life. Available literature indicates that the alloy behavior is independent of the flow rate (frequency of loading) when tested in the air environment and at various temperatures. However, $11 \%$ to $100 \%$ reduction in the fatigue strength at $10^{9}$ cycles was observed compared to that at standard $10^{6}$ cycles.

\section{Evaluation of creep properties}

The creep resistance of the nickel-based alloys depends on material grain structure. Two distinguished types of alloys are usually commercially available based on heat treatment and grain size: the high strength version with fine grain structure and the creep-resistant version with coarse grain structure. Depending on prevailing failure mechanism, creep or fatigue, material with fine or coarse grains are recommended.

In most cases, the creep data for bulk specimens is available in the form of the Larson-Miller parameter for rupture time and various levels of creep strains. The wall thickness effect (so-called thickness debit) on creep deformation and rupture properties of nickel-based alloys plays an important role in the durability analysis. A qualitative comparison of the thickness debit effect on the rupture life of differently manufactured superalloys was studied elsewhere. It was shown ${ }^{4}$ that a substantial reduction in creep life of the thin-walled castings compared with that of bulk castings occurs. One of the discussed causes of the thickness debit is the grain boundaries embrittlement caused by stress-assisted diffusion of the oxygen to the grain boundaries. However, this is not a case for the displacer dome, which is stressed in the helium environment and is nickel plated. 
In order to approximate the creep rupture properties for the thin-walled alloy, the Larson-Miller rupture time equation was generated from literature data. This approximated equation was used to evaluate the creep rupture for 148,920 hours resulting in rupture stresses being $146 \mathrm{MPa}$ and $115 \mathrm{MPa}$ at temperature of $820{ }^{\circ} \mathrm{C}$ and $840{ }^{\circ} \mathrm{C}$, respectively.

\section{Probabilistic Assessment of Hot Section Moving Components (based on displacer dome)}

A probabilistic analysis was performed in order to quantify uncertainties in the displacer dome structural response due to uncertainties in the design input variables. The input random variables include uncertainties in the thickness, the thermal and pressure loads, the thermomechanical material properties, the creep resistance, and fatigue strength. The predicted statistics in the structural responses are listed in Table 2. The additional source of uncertainties associated with the theoretical assumptions of the modeling and uncertainties estimations in the absence of enough test Table 2. Predicted statistic of the dome structural responses

\begin{tabular}{|l|c|c|l|}
\hline \multicolumn{1}{|c|}{ Output response } & Mean & CoV, \% & $\begin{array}{l}\text { Fitted probability } \\
\text { density function }\end{array}$ \\
\hline Dome radial expansion, $\mathrm{mm}$ & - & 5.1 & Normal \\
\hline Strain in dome section, $\%$ & - & 12.9 & Lognormal \\
\hline Strain in baffles, \% & - & 5.1 & Normal \\
\hline Stress in dome section, $\mathrm{MPa}$ & - & 11.7 & Lognormal \\
\hline Stress in cap section, $\mathrm{MPa}$ & - & 7.1 & Lognormal \\
\hline Stress in Baffles, MPa & - & 5.3 & Normal \\
\hline
\end{tabular}
data is not considered here.

Based on information available during this study, it is concluded that the reliability of the thin-walled displacer dome with brazed baffles made of nickel-based alloy is strongly dependent on scatter in the material strength. Also, when the coefficient of variation $(\mathrm{CoV})$ for fatigue strength is 30 $\%$ and lower, the material is capable of providing fatigue resistance under mission environment loads for the required mission duration of 17 years with a reliability of at least $99.12 \%$ as shown in Table 3 . It is found that uncertainties in the thin-walled fatigue and creep properties for the nickel-based alloy govern the hot section components reliability.

Table 4. Reliability of the dome section against creep

\begin{tabular}{|c|c|c|c|c|}
\hline \multirow{2}{*}{$\begin{array}{c}\text { Metal } \\
\text { temperature, }{ }^{\circ} \mathrm{C}\end{array}$} & \multirow{2}{*}{$\begin{array}{c}\text { Alloy creep } \\
\text { strength, } \\
\mathrm{MPa}\end{array}$} & \multirow[t]{2}{*}{$\mathrm{CoV}, \%$} & \multirow{2}{*}{$\begin{array}{l}\text { Distribution } \\
\text { function }\end{array}$} & Reliability, \% \\
\hline & & & & Dome section \\
\hline \multirow{3}{*}{820} & \multirow{3}{*}{ - } & 20 & \multirow{6}{*}{ Lognormal } & 100 \\
\hline & & 30 & & 99.77 \\
\hline & & 40 & & 98.27 \\
\hline \multirow{3}{*}{840} & \multirow{3}{*}{-} & 10 & & 100 \\
\hline & & 20 & & 99.87 \\
\hline & & 30 & & 98.09 \\
\hline
\end{tabular}

Additional knowledge of the material properties in the case of thickness debit effect could enhance the reliability prediction.

It is unlikely that the creep deformation will develop due to the lower temperature in the baffles and cylindrical section of the dome, for . For the dome section, the reliability against creep strongly depends on the material creep resistance at elevated temperature: when the $\mathrm{CoV}$ is below $20 \%$ the material is capable of providing creep resistance under mission environment loads for the required mission duration of 17 years with a reliability of at least $99.87 \%$ for the gas temperature of $840{ }^{\circ} \mathrm{C}$ as indicated in Table 4 .

The reported performance and reliability results were obtained using a simplified conservative analysis and may be validated more thoroughly with implementation of more rigorous deformation and material resistance model which require additional efforts. The remaining issues to investigate, which may enhance the study, are: (i) effect of the grain structure on long term response of the thin-walled alloys; (ii) effect of initial geometrical imperfections/roundness of the dome and hot cylinder; (iii) effect of launch vibration, that includes gas bearing properties in the simulation, on contact interference of the adjacent components.

\section{Reliability Analysis of Displacer Spring}

The displacer spring is another critical convertor component governing the life of the convertor. The displacer spring is subject to mechanical fatigue due to the reciprocating movement of the displacer assembly. In order to 
enhance the fatigue life of the displacer spring material, additional surface treatment using shot-penning is performed. This procedure benefits the material resistance to fatigue by developing the compressive residual stresses and hardening of the thin surface layers. In order to assess the fatigue strength of the shot-peened springs, it is necessary to develop a fatigue criterion which takes into consideration compressive residual stress and surface hardening.

The probabilistic method for reliability quantification of the displacer springs without surface treatment was reported earlier ${ }^{3}$. This method was updated for the surface treated springs. An extensive literature survey was conducted and a simplified analytical approach was developed to quantify the effect of the compressive residual stress on the fatigue strength of the shot-peened springs. Based on open literature review and available test data for a material similar to that of the spring, the following simplified relationship was adopted to evaluate the compressive residual stresses:

$$
\sigma_{s}^{r c} \approx A+1 / 2 \sigma_{\text {yield }}
$$

where $\sigma_{s}^{r c}$ is the compressive residual stress developed at the surface of the spring due to shot-peening, $\sigma_{\text {yield }}$ is the yield strength of the specific material, and $A$ is a parameter representing the overall effect of shot-peening. Although expression (6) is very simple, it was verified for many metals ${ }^{6}$ with an explicit value of parameter $A=120$ $M P a$. More refined finite element analysis combined with the results of the test data could be used to validate this approach.

7. Uncertainties in displacer spring design variables

Due to intrinsic uncertainties in material properties, in addition to uncertainty in the yield strength, parameter $A$ is also treated as uncertain assuming a normal distribution with the mean value of $A=120$ $M P a$. The large standard deviation of this variable would capture the possible relaxation of the compressive residual stress and the associated uncertainty. The described approach combined with the Goodman diagram ${ }^{6,7}$ was applied as a baseline for

Table 5. Uncertainties in the spring design variables

\begin{tabular}{|l|c|c|l|}
\hline \multicolumn{1}{|c|}{ Random variable } & $\begin{array}{c}\text { Mean } \\
\text { value }\end{array}$ & $\begin{array}{c}\text { Coefficient of } \\
\text { variation, \% }\end{array}$ & $\begin{array}{l}\text { Probability } \\
\text { Distribution }\end{array}$ \\
\hline Shot-peening parameter $\mathrm{A}, \mathrm{MPa}$ & - & 22.5 & Normal \\
\hline Yield strength, $\mathrm{MPa}$ & - & 7.5 & Lognormal \\
\hline Ultimate strength, $\mathrm{MPa}$ & - & 7.5 & Lognormal \\
\hline Unpeened fatigue strength, $\mathrm{MPa}$ & - & 24.0 & Lognormal \\
\hline
\end{tabular}
probabilistic prediction of the fatigue strength. Table 5 lists the uncertainties in the partial list of design variables used in this probabilistic analysis.

8. Summary of displacer spring reliability results

The results of the displacer probabilistic analysis are summarized as follows:

1) Reliability of the displacer spring against fatigue failure is predicted as 0.9913 .

2) Uncertainties in the un-peened fatigue strength, displacer stroke, and elastic modulus of the spring are key design factors governing the displacer spring reliability as shown in Fig. 1.

3) While displacer stroke could be controlled dynamically during the mission by a special sensor and controller, other design parameter variables have to be carefully chosen during the design stage. Incorrect usage of the shot-peening may affect the spring reliability.

4) Shot-peening parameter (SPP) affects the reliability of displacer spring non-linearly as shown in Fig. 2. The reliability of the displacer spring reduces with decreasing SPP. Therefore, possible relaxation of compressive residual stress during the mission may reduce the overall spring reliability.

5) Use of actual spring fatigue test data is required to validate the proposed approach and justify the fatigue strength of the shot-peened springs for operation up to fifty five billion cycles without failure. Upon receiving available information, the reliability analysis will be updated. 


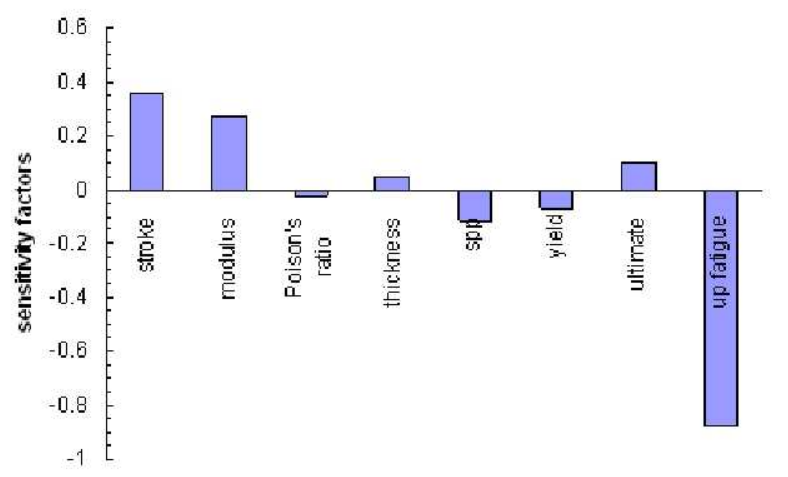

Figure 1. Sensitivity displacer spring reliability to design variables.

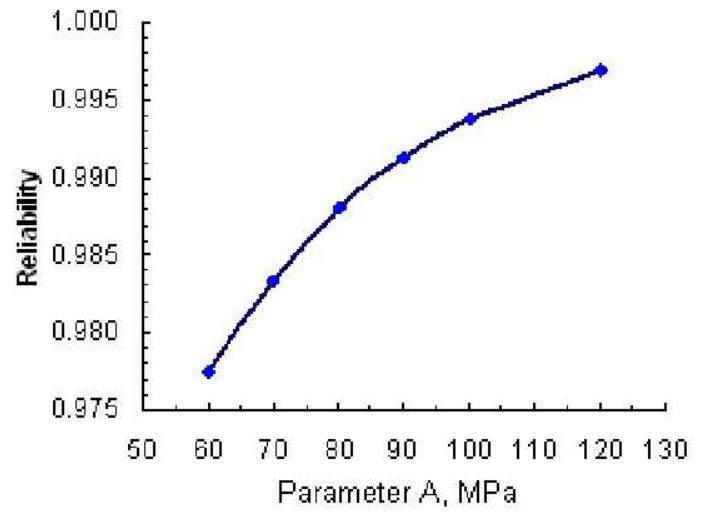

Figure 2. Effect of shot-peening parameter on displacer spring reliability.

\section{Reliability Analysis of ASC Subject to Vibration}

A random vibration analysis for the mission launch loads was performed in order to determine the convertor components responses during the launch. Due to vibration during the launch the structural stress/strains could amplified as well the component responses may be such that the required gap between the components may not be available for smooth operation of the convertor or may rub against each other. The launch vibration spectra in the form of the power spectral densities in axial and lateral directions prescribed in the NASA STD-7001 "Payload Vibroacoustic Test Criteria" were used as random excitation inputs to the ASC mounts. The reliability of the convertor components subjected to random base excitations was quantified with respect to exceedance of the specified thresholds at critical locations.

The lateral interaction between the moving and stationary components of the convertor is controlled by gas bearing by means of gas pressure in very narrow gaps. Ideally, a coupled fluid-structural analysis should be performed in order to account for the gas bearing restoring forces in the gaps during the entire Stirling cycle. The gas bearing analysis itself is complicated, requires additional computer resources, ${ }^{8}$ and become more complex when random loads are applied. One of the possible analysis simplifications is simulating the gas bearing restoring effect by elastic springs and viscous dampers. In this case, the springs and dashpots characteristics must be available. Presently, they are partially available for the flex-rod-piston-cylinder interfaces in the form of non-linear functions of the related gaps and were not implemented into the analysis. Instead, the following simplified two extreme conditions were modeled in order to establish the solution bounds, namely: (I) fully released contact with zero lateral stiffness and (ii) fully bounded lateral contact with infinite stiffness. The first extreme condition simulates the most possible soft contact with overclosure of the adjacent components, while no interference is allowed in the latter extreme.

9. Random vibration analysis method

As a prerequisite to the random vibration analysis, the modal analysis of the single convertor unit was performed. This analysis includes determining the convertor components natural frequencies and relevant mode shapes in the frequency ranges from $20 \mathrm{~Hz}$ to $2000 \mathrm{~Hz}$. By comparing the experimental vibrational spectra of the convertor with the computed resonant frequencies of the convertor internal parts, the most vibrational internal parts can be identified.

Random vibration analysis was performed using ANSYS computer code to compute statistics of each modal response and then combine using superposition technique to obtain component response statistics. The analysis assumed that the excitation is a stationary random process. Random loads in the form of power spectral densities were applied to the specified convertor mounts on the Advanced Stirling Radioisotope Generator, ASRG. The NASA qualification and flight acceptance spectra with overall level of 12.4 grms and 8.7 grms, respectively, were used in the analysis.

An attempt to simulate the lateral response of the gas bearings by means of the springs and dashpots in all frequency ranges was not computationally successful. Therefore, a simplified approach was used considering soft (lateral gas bearing stiffness is zero) and hard (lateral gas bearing stiffness is infinite) contact between the adjacent components. 
The response statistics in the form of the root mean square of the displacements and stresses of the convertor internal components were determined and results are summarized below:

1) Probable interference of the displacer dome and the hot cylinder is predicted in the case of the soft contact.

2) No contact between stator and pressure vessel is predicted.

3) Additional deflection of the displacer spring arms is predicted due to launch vibration. This introduces additional stresses in the displacer arms. The one-sigma of the predicted maximum equivalent von Mises stress is $56 \mathrm{MPa}$ and is located away from the critical stresses locations for the mission environment. These stresses are short in duration and have insignificant effect on life of the displacer spring.

4) The stresses in the heater head and transition arms due to launch vibration are very low compared to yield stress.

5) Attention must be paid to the predicted high stresses (Fig.3) in the flex rod, which supports the displacer components, causing rod bending. The probability of the stress in the flex rod exceeding the yield strength is 0.0129. It is understood, however, that the assumed soft contact, which neglects the gas

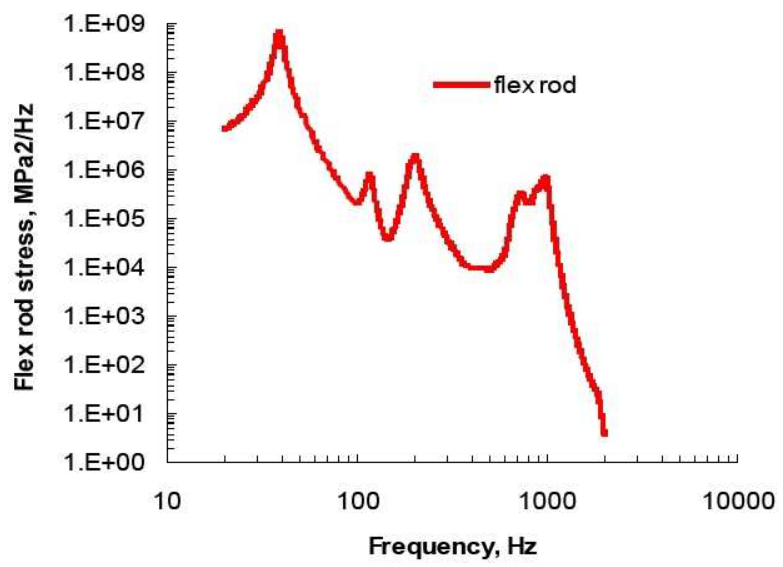

Figure 3. Spectral density of equivalent stress induced by launch vibration in flex rod.

bearing lateral support, is primary reason for this high stress. Therefore, more advanced modeling of the gas bearing restoring forces is recommended.

10. Reliability of the convertor critical components subject to random vibration

The root mean square (RMS) values of the displacements and stresses responses were used to compute the reliability of the components subject to launch vibration. Based on the results of the random vibration analysis with the qualification spectrum of 12.4 grms applied simultaneously in the axial $\mathrm{X}$ and lateral $\mathrm{Y}$ directions, the critical components were identified. Table 6 lists these components along with the probability of exceeding the corresponding material Table 6. Predicted convertor critical components at launch yield strength. It was assumed that the components mean stresses are zero and their standard deviations equal to the corresponding RMS.

\begin{tabular}{|c|c|c|c|c|}
\hline Components & $\begin{array}{c}\text { RMS, } \\
\mathrm{MPa}\end{array}$ & $\begin{array}{c}\text { Material Mean } \\
\text { Yield Strength, } \\
\mathrm{MPa}\end{array}$ & $\mathrm{CoV}, \%$ & $\begin{array}{c}\text { Probability } \\
\text { of failure }\end{array}$ \\
\hline Flex Rod & - & 758 & 2.5 & 0.0129 \\
\hline Transition arms & - & 1225 & 2.5 & $<0.0001$ \\
\hline Displacer spring & - & 470 & 6.5 & $<0.0001$ \\
\hline
\end{tabular}

\section{Physics-Based System Reliability Analysis of ASC}

Looking forward to future analysis, in order to implement the physics-based approach into the system reliability of the ASC, the following steps could be carried out. The first step would be the construction of a fault tree for the ASC. A fault tree is a logical and causal structure: a diagram of the convertor identifying the propagation of failure effects linked to the failure mechanisms at component level. The bottom level of the fault tree is composed of failure events at the component level. Failure events enable the occurrence of other intermediate events which ultimately lead up to the top-level event (convertor failure). The logical gates, OR-gate and the AND-gate are used as the primary logical gates connecting the bottom events to the top system level convertor failure event. It was learned that most analyzed failure mechanisms at the component level of the convertor lead to single point failures.

It is important and crucial to note here that we propose the idea that a physics-based probabilistic fault tree analysis (PFTA) for the ASC can be performed that is somewhat different than a conventional fault tree analysis. Although both approaches might appear to be similar there are glaring significant differences in the way the 
uncertainties would be simulated and propagated from bottom level (basic events) to the top level (system) event. The bottom level component blocks in the PFTA tree specify a symbolic set of cumulative distribution functions $(\mathrm{CDFs})$ for the component performance and failure that is directly related to physical design parameters or physical variables. The $\mathrm{x}$-axis of each $\mathrm{CDF}$ in the set is a value of the limit-state function, $\mathrm{G}$, which is a difference between the resistance-to-failure, $\mathrm{R}$, and the load effect, S. (e.g. $G=R-S$ ). The threshold for failure is when $\mathrm{G}<0$, or the stress exceeds the resistance-to-failure. The probability of failure is the probability that $\mathrm{G}<0$. This relationship and inter-relationship among the components especially is governed by common component and system design variables and is retained during the system reliability simulation throughout. This is how the PFTA differs significantly as compared to the conventional approach. The PFTA enables quantification of the system reliability and how the reliability is sensitive to design variables at the bottom level of the tree. The limit state functions of each bottom event in the fault tree, and associated CDFs would be determined using the results of the physics-based probabilistic analysis at the component level. The convertor system reliability would be quantified using an adaptive importance sampling method and direct Monte Carlo simulation.

In the current development stage, convertor system probabilistic models include the combination of failure modes related to creep of the heater head, creep and fatigue of the displacer dome, giga-cycle fatigue of the displacer spring and check valve, and fibers breakage of the random-fibers within the regenerator. Twenty nine random variables are included in probabilistic models.

The Monte Carlo simulation can be used to predict the system reliability and sensitivity to the random variables.

\section{Conclusion}

Physics-based reliability analysis is being performed to assure that the Advanced Stirling Convertor (ASC) can provide power for a deep-space mission for as long as 17 years with desired performance, efficiency and reliability. Computer simulation with limited test data verification showed to be a viable approach to assess the reliability of ASC components. Physics-based reliability analysis involved the relationship among the design variables based on physics, mechanics, material behavior models, interaction of different components and their respective disciplines such as structures, materials, fluid, thermal, mechanical, electrical, etc. The failure mechanisms and causes of failure of the convertor components were studied, especially in light of the new information, in order to develop guidelines to improve design reliability and better controls for reducing the probability of failure. Quantified reliability assessment based on fundamental physical behavior of components and their relationship with other components is a superior technique to conventional reliability approaches based on utilizing failure rates derived from similar equipment or simply expert judgment.

\section{Acknowledgments}

This work is funded through the National Aeronautics and Space Administration (NASA) Science Mission Directorate and was supported by the NASA Glenn Research Center. Any opinions, findings, and conclusions or recommendations expressed in this article are those of the authors and do not necessarily reflect the views of NASA. The authors also appreciate and thank the program management at GRC provided by Dick Shaltens and Gary Kelm. We thank Jeff Schreiber, Wayne Wong, and Chuong Ha (at Lockheed-Martin) for their technical insights.

\section{References}

\footnotetext{
${ }^{1}$ Shah, A. R., Schreiber, J. G., "Reliability Issues in Stirling Radioisotope Power Systems, " NASA TM-2004-213428, 2004.

${ }^{2}$ Brinkman, C. R., Booker, M. K., and Ding J. L., "Creep and Creep-Rupture Behavior of Alloy 718. Superalloys 718,625 and Various Derivatives, Edward A. Loria, ed., The Minerals, Metals, Materials Society, Warrendale, PA, 1991, pp. 519-536.

${ }^{3}$ Shah, A. R.,et al, "Reliability Quantification of the Flexure: A Critical Stirling Convertor Component," Proc. Ann. Reliability \& Maintainability Symp., (Jan.) 2005, pp 270-275.

${ }^{4}$ Epishin T., "Mechanisms of High Temperature Creep of Nickel-Based Superalloys Under Low Applied Stress, "Superalloys 2004, TMS, 2004, pp.137-143.

${ }^{5}$ Wang,S.,Li,Y.,Yao,M.,Wang,R., "Compressive residual stress induced by shot peening," Journal of Mat. Proc.Tech., 1998, 73, 64-73.

${ }^{6}$ Bannantine J. A.,et al, "Fundamentals of Metal fatigue Analysis, ” New Jersey, Prentice Hall, 1990.

${ }^{7}$ Stephens R.I, et al., "Metal fatigue in Engineering, "New York, J.Wiley, 2001.

${ }^{8}$ Dyson, R. W. “Analysis - Gas Bearings \& Centerport, ”, ASC-E2 Critical Design Review, July 8-10, 2008, pp.418-442.
} 